\title{
Quality Choice, Fixed Costs and Equilibrium in Models of Vertical Differentiation $^{1}$
}

\author{
Matteo Alvisi ${ }^{2}$ \\ April 2000 \\ University of Bologna \\ Department of Economics
}

\begin{abstract}
I provide a full characterization of the quality choice in duopolies with vertical differentiation, without assuming ex-ante if the market is fully covered or not. This will allow to show that covered or uncovered market configurations are endogenous outcomes of firms' strategic interaction. To this purpose, I assume that firms are characterized by quadratic fixed costs of quality improvements and check whether pure-strategy subgame perfect equilibria with a corner solution always exist. Finally, my results are compared to the quality choice that maximizes the total surplus of the economy. I show that the welfaremaximizing choice of qualities does not have to be found in the same market configuration of the corresponding market equilibrium.
\end{abstract}

Keywords: quality, oligopoly, vertical differentiation, market configuration, subgame perfect equilibrium, welfare analysis.

\footnotetext{
${ }^{1}$ I would like to thank David Levine for his constant encouragement and suggestions. I am also grateful to Thomas Hubbard, Harold Demsetz, Carlo Scarpa and all seminar participants at the University of California, Los Angeles for their useful comments.

${ }^{2}$ Department of Economics, UCLA and University of Bologna.

E-mail: malvisi@economia.unibo.it.
} 


\section{Introduction}

Oligopolies in which firms sell products of different qualities have been analyzed first in Gabszewicz and Thisse (1979). They concluded that price competition could yield either market outcomes where some consumers prefer to refrain from buying (uncovered market configuration) or outcomes where all consumers buy one of the two products (covered market configuration). The alternative which actually realizes depends on the degree of product differentiation and the extent of consumers' heterogeneity ${ }^{3}$. Assuming that firms do not cover the market, Choi and Shin (1992) show that the low quality firm will choose a quality level which is a fixed proportion of the higher quality's firm choice. On the contrary, Tirole (1988) assumes that firms cover the market and shows that firms maximize product differentiation over the available range of qualities. In this discussion, we attempt first to provide a full characterization of the quality choice, without assuming ex ante that the market is, or is not, covered. This will allow us to show that covered or uncovered market configurations are endogenous outcomes of firms' strategic interaction. The first who recognized this possibility is Wauthy (1996), analyzing a vertically differentiated duopoly where firms produce at no cost. He identifies the parameter ranges where either a covered or an uncovered market configuration arises in equilibrium, as well as a range where a corner solution at the price stage is obtained. Ecchia e Lambertini (1998) study a similar model with quadratic variable costs of quality improvements, so that the well known finiteness property does not hold (see Shaked and Sutton, 1983, Crampes and Hollander, 1995). They investigate the existence and characterization of pure strategy subgame perfect equilibria for a fixed market size, considering alternatively both the uncovered and covered market configuration. Similarly to Wauthy, they find a parameter range in which neither configuration can be properly defined; however, when unit costs of investment in quality are introduced, they prove that a pure strategy equilibrium which contains a corner solution in the price subgame fails to exist. This is due to the incentive for the high-quality firm to set a quality such that the rival's sales are driven to zero.

We consider a vertically differentiated oligopoly with quadratic fixed costs of quality improvements (so that, similarly to Shaked and Sutton's contribution, the finiteness property holds), analyzing whether in this case pure-strategy subgame perfect equilibria with a corner solution in the price subgame exists. 
Contrarily to Wauthy, in our model the high-quality firm faces a trade-off in increasing its quality: higher product differentiation might lead to higher equilibrium prices, but at the cost of an higher investment in quality and possibly of a lower market share.

With respect to Lambertini and Ecchia (1998), we also find parameter ranges in which pure-strategy equilibria don't exist. The presence of fixed costs, however, allows equilibria with corner solutions and possibly the disappearance of equilibria will full coverage. Also, we compare our results to the quality choice that maximizes the total surplus of the economy. Contrarily to what happens in the previous literature on the subject, we will show that the welfare-maximizing choice of qualities for a given distribution of consumers' characteristics doesn't have to be found in the same market configuration of the equilibrium.

In the next section we will set up the model. In Section 3 we derive the equilibrium in the price subgame, while in Section 4 quality choice will be discussed. Section 5 derives the welfare-maximizing choice of quality levels and Section 6 presents some concluding remarks.

\section{The model}

The basic features of the model used are standard in studies of quality differentiation with oligopolistic competition (Mussa and Rosen, 1978, Gabszewicz and Thisse, 1979, Shaked and Sutton, 1982, Ronnen, 1991 and Constantatos and Perrakis, 1998). The demand side of the market consists of a continuum of consumers indexed by $s$, the taste parameter ${ }^{4}$. We assume that $s$ is continuously and uniformly distributed on an interval $[b, a]$, where $a, b>0$. For simplicity, we normalize the difference between the extremes of the distribution to 1: $a-b=1^{5}$. Let $u\left(s, p_{i}, \boldsymbol{\theta}_{i}\right)=\max \left[0, s \boldsymbol{\theta}_{i}-p_{i}\right]$ be the utility function for each consumer, where $\theta_{i}>0$ is the quality of the product sold by firm $i$ and $p_{i}$ the corresponding price. The implicit assumption is that each consumer buys at most one unit of services. In case of no purchase, the utility would be zero (notice that the assumption of unit purchase seems realistic when dealing with professional services).

\footnotetext{
${ }^{3}$ The intuition that firms are led to choose different qualities in equilibrium has been further investigated by Shaked and Sutton (1982).

${ }^{4}$ It would be equivalent to assume that consumers differ on income, instead of tastes.

${ }^{5}$ This normalization implies that $a>1$.
} 
There are two potential entrants to the market, identical in all aspects. In particular, each of them is constrained to offer only one quality, and faces the same fixed costs of developing the technology that enables the provision of quality $\theta$. These quality-dependent fixed costs, $C(\theta)$, and their respective marginal costs $C^{\prime}(\theta)$, are assumed to be increasing with $\theta . C^{\prime}(\cdot)>0$ and $C^{\prime \prime}(\cdot)>0$ for all feasible qualities $\theta \in[0, \infty)$. Throughout the analysis we shall also maintain the following regularity assumptions: $C(0)=C^{\prime}(0)=0$ and that $\lim _{\theta \rightarrow \infty} C^{\prime}(\theta)=\infty$. Marginal costs of production are assumed to be constant (without loss of generality we suppose they are equal to zero $)^{6}$.

Competition between the two firms is based on a three stage non-cooperative game. In the first stage, firms choose whether or not to enter the industry. At the end of the first stage, each firm observes who entered and who didn't. In the second stage each firm chooses the quality of its service. Then, having observed its rivals' qualities, in the final stage of the game, each firm chooses its price. These assumptions are intended to capture the idea that the price can in practice be varied at will, but a change in the specification of the service involves modification of the appropriate "production facilities" (Shaked and Sutton, 1982).

Consumers are price and quality takers: given the two firms' decisions, $\left(\boldsymbol{\theta}_{i}, p_{i}\right)_{i=1,2}$, each consumer chooses between purchasing one unit from firm 1, purchasing one unit from firm 2, or making no purchase.

The solution concept we employ is (pure-strategy) subgame perfect equilibrium. First, note that if only one firm entered the market in the first stage, it becomes a monopolist in the third; in this case, it is easy to verify that, if $a<2, p_{M}=(a / 2) \boldsymbol{\Theta}_{M}$, while the monopoly market share is $[a / 2, a]^{7}$.

Otherwise, if both firms enter the market, then the second-stage subgame is characterized by a couple $\left(\boldsymbol{\theta}_{1}, \boldsymbol{\theta}_{2}\right) \in[0, \infty) \times[0, \infty)$; however, if the same qualities are set, then the services become homogeneous and Bertrand competition in the third stage would drive profits to zero. In models of vertical differentiation then, only the asymmetric case of different qualities has to be considered, since there is no profitable deviation consisting in setting equal qualities. In this respect, the strategies of the second stage could be equivalently defined as $\left(\boldsymbol{\theta}_{H}, \boldsymbol{\theta}_{L}\right), \boldsymbol{\theta}_{H} \geq \boldsymbol{\theta}_{L}$, where the subscript $H$ denotes the firm that has entered the market with a

\footnotetext{
${ }^{6}$ This seems to be a consistent assumption with the industry of professional services (see the second part of this analysis). The main cost for lawyers, physicians, architects and so on is the fixed cost they have to pay for their education period (in general terms, the longer their education, the higher the quality of the service that they can provide). Instead, it does not seem that unit costs depend on quality (variable costs are thus constant).

${ }^{7}$ If $a<2$, the monopoly will then serve only part of the market and the equilibrium will result in an "uncovered configuration". Later in the paper, we will in fact assume $a<2$, in order to avoid the analysis of the "preemption" case.
} 
quality that exceeds the one of the other firm (denoted with $L$ ); the former (latter) firm will be labeled the high-quality (low-quality) firm ${ }^{8}$.

\section{Price competition}

We will now focus on the Nash equilibrium of the third stage of the game in which both firms are active. In this price subgame, qualities $\left(\theta_{H}>\theta_{L}>0\right)$ are exogenous and in the equilibrium the two firms will obviously set prices such that $p_{H}>p_{L}{ }^{9}$.

Demand addressed to firm $i(i=L, H)$ is defined by the set of consumers who maximize utility when buying product $i$ rather than product $j$ or refraining from buying. Given $\left(p_{H}, p_{L}\right)$, we denote by $\bar{s}=\bar{s}\left(p_{H}, p_{L}\right)$ the marginal consumer who is indifferent between consuming either one of the two products and by $\underline{s}$ the consumer with the lower taste for quality who however still buys one unit of the (low-quality) product, that

is $\bar{s}=\frac{p_{H}-p_{L}}{\theta_{H}-\theta_{L}}$ and

$\underline{s}= \begin{cases}\frac{p_{L}}{\boldsymbol{\theta}_{L}} & \text { if } \frac{p_{L}}{\boldsymbol{\theta}_{L}}>b \\ b \quad \text { otherwise } & \cdot^{10}\end{cases}$

In equilibrium some consumers may refrain from buying, as indicated in (1a). In this case we say that the market is characterized by an uncovered configuration; otherwise we say that the market is covered. Finally, since $\boldsymbol{\theta}_{H}>\boldsymbol{\theta}_{L}$, all consumers would prefer to use the high quality service if $p_{H}=p_{L}$. The high-quality firm may thus benefit from the possibility of preempting the market with a limit price, $p_{H}^{l}=p_{L}+b\left(\boldsymbol{\theta}_{H}-\boldsymbol{\theta}\right.$ L). This implies that, as pointed out originally by Gabszewicz and Thisse (1979), three market

\footnotetext{
${ }^{8}$ Note that the two firms are ex-ante identical. If a subgame perfect equilibrium exists with firm 1 being the high-quality firm, then a totally symmetric equilibrium with firm 2 being the high-quality also exists. From now on, however, we will only label firms with $H$ and $L$.

${ }^{9}$ In fact, if $p_{H} \varsigma_{L}$ the low-quality firm would have no market share and overall (considering the quality-dependent fixed costs suffered in the second stage for $\boldsymbol{\theta}_{L}$ ) would get negative profits.

${ }^{10}$ By definition, $\bar{s}$ satisfies $\bar{s} \Theta_{H}-p_{H}=\bar{s} \Theta_{L}-p_{L}$. Accordingly, consumers with $s>(<) \bar{s}$ strictly prefer the high(low)-quality product. Similarly, $\underline{s}$ is defined in (1a) such that $\underline{s}_{L}-p_{L}=0$ so that if $s<\underline{s}$ the consumer will refrain from buying,
} 
configurations may arise at the price equilibrium and they are characterized by the following demand functions $\left(p=\left(p_{H}, p_{L}\right)\right)$ :

(1) uncovered market: $D_{H}(p)+D_{L}(p)<1, D_{i}>0, i=L, H$,

$D_{H}(p)=\left(a-\frac{p_{H}-p_{L}}{\theta_{H}-\theta_{L}}\right)$,

$D_{L}(p)=\left(\frac{p_{H}-p_{L}}{\boldsymbol{\theta}_{H}-\boldsymbol{\theta}_{L}}-\frac{p_{L}}{\boldsymbol{\theta}_{L}}\right)$

(2) covered market: $D_{H}(p)+D_{L}(p)=1, D_{i}>0$,

$D_{H}(p)=\left(a-\frac{p_{H}-p_{L}}{\boldsymbol{\theta}_{H}-\boldsymbol{\theta}_{L}}\right)$

$D_{H}(p)=\left(\frac{p_{H}-p_{L}}{\boldsymbol{\theta}_{H}-\boldsymbol{\theta}_{L}}-b\right)^{11}$

(3) Preempted market: $D_{H}(p)=1, D_{L}(p)=0$.

The Nash equilibrium in the price game is obtained in two steps. Given $a, \theta_{H}$ and $\theta_{L}$ we first compute the equilibrium candidates for each market configuration. Then, we identify the parameters constellations such that these candidates effectively yield the corresponding market outcome. In particular, we will identify four intervals for the value of $d_{p}=a / b=a /(a-1)$, a rough index of consumers' heterogeneity, whose bounds will depend on the degree of product differentiation, i.e. on $d_{q}=\theta_{H} / \theta_{L}$ :

\subsection{Uncovered market configuration}

In this case, the revenues of the two firms are

$$
T R_{H}(p)=p_{H}\left(a-\frac{p_{H}-p_{L}}{\boldsymbol{\theta}_{H}-\boldsymbol{\theta}_{L}}\right) ; \quad T R_{L}(p)=p_{L}\left(\frac{p_{H}-p_{L}}{\boldsymbol{\theta}_{H}-\boldsymbol{\theta}_{L}}-\frac{p_{L}}{\boldsymbol{\theta}_{L}}\right)
$$

and the "relevant" portion of the best response functions is ${ }^{12}$ :

\footnotetext{
${ }^{11}$ Note that while the high-quality firm market share is the same in both covered and uncovered configurations, this doesn't happen for the low-quality firm. Only in the first case the low-quality firm can modify the lower limit of its share with a proper choice of $p_{L}$ and $\boldsymbol{\Theta}_{L}$.
} 
$p_{H}\left(p_{L}\right)=\frac{a\left(\boldsymbol{\theta}_{H}-\boldsymbol{\theta}_{L}\right)+p_{L}}{2} ; \quad p_{L}\left(p_{H}\right)=\frac{p_{H} \boldsymbol{\theta}_{L}}{2 \boldsymbol{\theta}_{H}}$

so that the equilibrium prices are:

$$
p_{H}^{*}=\frac{2 a \boldsymbol{\theta}_{H}\left(\boldsymbol{\theta}_{H}-\boldsymbol{\theta}_{L}\right)}{4 \boldsymbol{\theta}_{H}-\boldsymbol{\theta}_{L}} ; \quad p_{L}^{*}=\frac{a \boldsymbol{\theta}_{L}\left(\boldsymbol{\Theta}_{H}-\boldsymbol{\theta}_{L}\right)}{4 \boldsymbol{\theta}_{H}-\boldsymbol{\theta}_{L}}
$$

Notice that both equilibrium prices depend positively on $a$ (and then negatively on $d_{p}$ ): the "more" consumers value quality, the greater the revenues for both firms, and this preference will be then reflected on prices. Also, the high-quality firm's share is always twice as high as the low-quality firm's share and both increase with $a$ :

$\left(a-\frac{p_{H}^{*}-p_{L}^{*}}{\boldsymbol{\theta}_{H}-\boldsymbol{\theta}_{L}}\right)=2 a \frac{\boldsymbol{\theta}_{H}}{4 \boldsymbol{\theta}_{H}-\boldsymbol{\theta}_{L}} ; \quad\left(\frac{p_{H}^{*}-p_{L}^{*}}{\boldsymbol{\theta}_{H}-\boldsymbol{\theta}_{L}}-\frac{p_{L}^{*}}{\boldsymbol{\theta}_{L}}\right)=a \frac{\boldsymbol{\theta}_{H}}{4 \boldsymbol{\theta}_{H}-\boldsymbol{\theta}_{L}}$,

implying that as $a$ increases, so that consumers' preferences are more concentrated, the share of "excluded" consumers decreases. Finally, it is noteworthy that $\frac{\partial p_{L}^{*}}{\partial \boldsymbol{\theta}_{L}}>0$ only if $\boldsymbol{\theta}_{L}$ is sufficiently small: intuitively, increasing $\theta_{L}$ would make the two services closer substitutes and push $p_{H}$ and $p_{L}$ down. However, as $\theta_{L}$ increases, also the low-quality firm's market share increases and this effect might prevail.

This price equilibrium in an uncovered configuration exists however only if, as assumed, $D_{H}\left(p^{*}\right)+D_{L}\left(p^{*}\right)<1$ or equivalently, only if

$$
d_{p} \geq \frac{4 d_{q}-1}{4 d_{q}-2}
$$

\subsection{Covered market configuration}

If the values of $a, \theta_{H}$ and $\theta_{L}$ are such that (5) is not satisfied there might be however a price equilibrium with a covered configuration, where all consumers purchase the service. In this case the revenue and the

\footnotetext{
${ }^{12}$ These are restricted best responses, because for a relatively low value of $p_{H}$, the true best response of the low-quality firm is to play as the high-quality firm with some $p_{L} \in\left(p_{H}, \infty\right)$. For the complete version of the best response functions and the uniqueness of the price equilibrium in the uncovered configuration when both firms are active, see Ronnen (1991).
} 
"relevant" portion of best response function for the high-quality firm are the same as in section $3.1^{13}$, while for the low-quality firm they are equal, respectively, to

$$
T R_{L}(p)=p_{L}\left(\frac{p_{H}-p_{L}}{\boldsymbol{\theta}_{H}-\boldsymbol{\theta}_{L}}-(a-1)\right) ; \quad p_{L}\left(p_{H}\right)=\frac{1}{2}\left(p_{H}-(a-1)\left(\boldsymbol{\theta}_{H}-\boldsymbol{\theta}_{L}\right)\right)
$$

so that the equilibrium prices are:

$$
p_{H}^{* *}=\frac{(a+1)\left(\boldsymbol{\theta}_{H}-\boldsymbol{\theta}_{L}\right)}{3} ; \quad p_{L}^{* *}=\frac{(2-a)\left(\boldsymbol{\theta}_{H}-\boldsymbol{\theta}_{L}\right)}{3}
$$

Notice that in this price equilibrium $p_{L}^{* *}$ depends negatively on $a$. The positive effect described for the uncovered configuration is dominated by a decrease in the low-quality firm's market share which pushes its price down. In fact, the low-quality firm's share decreases with $a$ and doesn't depend on the two firms' qualities:

$$
\left(\frac{p^{* *}{ }_{H}-p^{* *} L}{\theta_{H}-\theta_{L}}-(a-1)\right)=\frac{2-a}{3} .
$$

Moreover, $\frac{\partial p^{* *}{ }_{L}}{\partial \theta_{L}}<0$ : increasing $\theta_{L}$ makes the two products closer substitutes and always pushes $p_{L}$ (and $p_{H}$ ) down. As it happened in section 3.1, however, this equilibrium is defined only if at these prices we are indeed in a covered configuration, as assumed. In particular, the price set by the low-quality firm should be positive, $a<2$, while the consumer with the lower taste for quality should enjoy a non-negative utility by purchasing the (low-quality) service,

$$
(a-1) \boldsymbol{\theta}_{L} \geq p_{L}^{* *}
$$

in other terms

$$
\frac{2 d_{q}+1}{d_{q}-1} \geq d_{p}>2 .
$$

\subsection{Corner solution and preemption}

C) If the value of $d_{p}$ is such that not even the above condition is satisfied, that is if

$$
\frac{4 d_{q}-1}{4 d_{q}-2} \leq d_{p} \leq \frac{2 d_{q}+1}{d_{q}-1},
$$

\footnotetext{
${ }^{13}$ See previous footnote.
} 
still an equilibrium with both firms operating in the market exists and it is characterized by a corner solution: the high-quality firm's best response function is the same as in (2), while the low-quality firm quotes the price which is just sufficient to cover the market. The equilibrium prices in this case are then:

$$
p_{H}^{c}=\frac{a \boldsymbol{\theta}_{H}-\boldsymbol{\theta}_{L}}{2} ; \quad p_{L}^{c}=(a-1) \boldsymbol{\theta}_{L}
$$

In the corner configuration, $p_{L}$ depends positively on $\theta_{L}$ and this appears counterintuitive. However, note that an increase of $\theta_{L}$ implies a relaxation of the constraint (8) and allows the low-quality firm to set an higher price, closer to the unconstrained "optimum". Also, while the low-quality firm's share depends positively on $d_{p}$ as in the covered configuration, it also depends on the (exogenous) qualities chosen in the second stage, as in the uncovered configuration. Clearly, as $d_{p}$ increases, the transition from an uncovered to a covered configuration is not smooth: for a significant range of this parameter, as Wauthy (1996) originally underlined, the price equilibrium is a corner solution. The relevance of the result is due to the fact that the nature of competition in this three configurations is different. When the market is covered, in fact, price competition is a pure battle for market shares, while in the other two configurations part of this battle is played during the second stage, as well, even if with different implications ${ }^{14}$. As product differentiation increases, the low-quality firm's share decreases in the uncovered configuration, because the increase in $p^{*_{H}}$ is counterbalanced not simply by an increase in $p^{*}$ but also by a greater amount of "excluded consumers". On the contrary, as long as $d_{p}$ and $d_{q}$ are such that a corner solution is defined, the market is totally covered, so that greater quality differentiation is a clear advantage for the low-quality firm.

If $d_{p}<2$ the market will be preempted by the high-quality firm. The equilibrium is given by $p^{P}{ }_{L}=0$ and $p^{P}{ }_{H}$ $=(a-1)\left(\theta_{H}-\theta_{L}\right)$. However, our interest is focused on situations in which both firms are active, so that from now on we will assume that $d_{p}>2$.

In conclusion, unique price equilibria exist in every configuration; there is clearly an intrinsic advantage for the high-quality firm, which in every configuration enjoys higher revenues ${ }^{15}$. Also, in any equilibrium price

\footnotetext{
${ }^{14}$ The share for the low-quality firm in the covered configuration is $1-\frac{(a-1) \boldsymbol{\theta}_{H}}{\boldsymbol{\theta}_{H}-\boldsymbol{\theta}_{L}}$.

${ }^{15}$ It is easy to verify that in every configuration, a price strategy for each type of firm that makes the other firm's share equal to 0 implies for the low-quality firm a price well under its rival's price, while the opposite would happen for the high quality firm: using its better quality the latter can "eliminate" the other product while maintaining an higher price. The
} 
competition intensifies as the quality differentiation shrinks since then the two products become closer substitutes. Finally, it is the relative position of $d_{p}$ and $d_{q}$ which determine the relevant price equilibrium:

Proposition 1: The Nash equilibrium in prices and the associated market outcomes are determined as a function both of population heterogeneity and the degree of product differentiation ${ }^{16}$.

Note that only $d_{p}$ is exogenous to the model. Qualities will be optimally chosen by both firms in the second stage of the game. In the next section we will show how the market configuration is in fact endogenous to the model; in their choice of quality, firms will decide in which "environment" they want to play and this will influence both the degree of market coverage and the nature of price competition in the last subgame.

\section{The quality choice}

Finding a perfect equilibrium for the second-stage game in which quality is chosen, and then price, is equivalent to find a Nash equilibrium in qualities. The payoffs arise from any couple of qualities being defined by the (unique) equilibrium couple of revenues in the "price choice" of the previous section.

Depending on the configuration, the total revenues arising from the price equilibrium are the following:

(A) Uncovered configuration: $T R_{H}^{*}=\frac{4 \boldsymbol{\theta}_{H}^{2}\left(\boldsymbol{\theta}_{H}-\boldsymbol{\theta}_{L}\right)}{\left(4 \boldsymbol{\theta}_{H}-\boldsymbol{\theta}_{L}\right)^{2}} ; \quad T R_{L}^{*}=\frac{\boldsymbol{\theta}_{L} \boldsymbol{\theta}_{H}\left(\boldsymbol{\theta}_{H}-\boldsymbol{\theta}_{L}\right)}{\left(4 \boldsymbol{\theta}_{H}-\boldsymbol{\theta}_{L}\right)^{2}}$;

(B) Covered configuration: $T R_{H}^{* *}=\frac{(a+1)^{2}\left(\boldsymbol{\theta}_{H}-\boldsymbol{\theta}_{L}\right)}{9} ; \quad T R_{L}^{* *}=\frac{(a-2)^{2}\left(\boldsymbol{\theta}_{H}-\boldsymbol{\theta}_{L}\right)}{9}$;

(C) $\underline{\text { Corner solution: }} T R_{H}^{C}=\frac{\left(a \boldsymbol{\theta}_{H}-\boldsymbol{\theta}_{L}\right)}{4\left(\boldsymbol{\theta}_{H}-\boldsymbol{\theta}_{L}\right)} ; \quad T R_{L}^{C}=\frac{(a-1) \boldsymbol{\theta}_{L}\left((2-a) \boldsymbol{\theta}_{H}-\boldsymbol{\theta}_{L}\right)}{2\left(\boldsymbol{\theta}_{H}-\boldsymbol{\theta}_{L}\right)}$.

incentive in pursuing this strategy is then different between the two firms See Lemma 3 in Shaked and Sutton (1982) and Polo (1991) for the covered and uncovered case, respectively.

${ }^{16}$ See Gabszewicz and Thisse (1979) for a slightly different version of this result. 
In the absence of any quality-dependent fixed costs the Nash equilibrium strategy for the high-quality firm is quickly determined; independently of the market configuration, $T R_{H}$ depends positively on $\theta_{H}$, so that if we assume that there is a maximum quality $\overline{\boldsymbol{\theta}}$ that each firm can produce, then $\theta_{H}=\overline{\boldsymbol{\theta}}$. Shaked and Sutton (1982) examined this case considering a covered market only (cases B and C), while Wauthy (1996) pointed out more clearly that the choice of the low-quality firm depends on the population characteristics. In his contribution, without quality-dependent fixed costs, one firm chooses the best available quality and the other firm either chooses a fixed proportion of the best quality or a quality level which is determined by population characteristics. This determines whether the market is covered or not in the price game.

Our analysis welcomes the idea that the distribution of consumers' tastes is a decisive factor for product differentiation. When the distribution is broad, we expect that quality choices will result in the market being served partially, while when it is concentrated, a fiercer price competition cannot be avoided by the choice of qualities. However, this result clearly depends on the absence of any cost in producing quality, the main implication of it being the elimination of any strategic element in the high-quality firm's behavior. Also, this hypothesis always insures the existence and uniqueness of the Nash equilibrium in qualities: with the highquality firm setting its quality at the highest possible level, the-low quality firm can actually choose the configuration that brings it the highest profits.

If we recognize that investing in quality is $\operatorname{costly}^{17}$, results might change: (1) (pure strategy) Nash equilibria in qualities might not always exist; (2) there are conditions which eliminate the possibility of a particular market configuration to actually emerge in equilibrium; (3) while the equilibrium in qualities is always suboptimal, it is not clear whether, for a given distribution of consumers' tastes, the comparison with the social optimum should be performed conditional on the equilibrium market configuration. Even if we wanted to restrict our attention to one particular configuration (as previous analyses often do), we need to recognize that the social optimum might imply a different market structure.

Finally, the absence of quality costs is not particularly satisfying for regulation purposes. Consider for instance the introduction of price floors with the goal of increasing the average level of quality. Without costs, the high-quality firm's behavior wouldn't change and the only effect would be an increase in the

\footnotetext{
${ }^{17}$ In the example of professional services, the cost of the investment in human capital seems indeed one of the most important factors influencing the professionals' strategic behavior.
} 
quality chosen by the other firm ${ }^{18}$; the conclusion is that price floors would be always welfare-improving. In the remaining of this paper, then, we analyze what happens when we assign a more active strategic role to the high-quality firm in its choice of quality.

\subsection{Fixed costs and quality choice}

For a (pure strategy) Nash equilibrium in qualities to exist it must be that each player is playing his best response; also, if it exists, it must be in one of the three configurations defined in Section 3. Our procedure will then follow the following steps:

i) we obtain the equilibrium candidates in qualities for a given market configuration by looking at both firms' best response function;

ii) we check for which values of the parameter $d_{p}$ these equilibria are defined and if there exist sets of values for this parameter such that more than one configuration is feasible;

iii) we check if, given part i), at least one of the two firms has any incentive to deviate by setting a quality which would lead to a price subgame in a different configuration;

iv) we define the subgame perfect Nash equilibrium for different values of $d_{p}$.

In order to find closed-form solutions at least for the equilibrium of the first two market configurations, it seems appropriate to assume here a specific functional form for the cost function. From now on, then,

Assumption 1: $C(\theta)=\frac{1}{2} \theta^{2}$.

\subsection{Partial market coverage}

Focusing first on the uncovered configuration, Ronnen (1991) proved the existence and uniqueness of the equilibrium :

Theorem 1 (a) There exists a unique pair $\left(\theta^{*}{ }_{H}, \theta^{*}\right)$ in the uncovered configuration which satisfies the following two conditions:

\footnotetext{
${ }^{18}$ When the price floor is binding (so that the low-quality firm has to increase its price above the market equilibrium level, independently of the market configuration), the low-quality firm clearly reacts by increasing its quality in order not to lose its market share.
} 
$M R_{L}\left(\boldsymbol{\theta}_{H}, \boldsymbol{\theta}_{L}\right)=M C\left(\boldsymbol{\theta}_{L}\right), \boldsymbol{\theta}_{L} \in\left(0, \boldsymbol{\theta}_{H}\right)$ and $M R_{H}\left(\boldsymbol{\theta}_{H}, \boldsymbol{\theta}_{L}\right)=M C\left(\boldsymbol{\theta}_{H}\right), \boldsymbol{\theta}_{H} \in\left(\boldsymbol{\theta}_{L}, \infty\right) ;$

(b) Both firms earn positive profits when $\left(\theta^{*}{ }_{H}, \theta^{*}\right)$ is played;

(c) $C^{\prime \prime \prime}(\cdot) \geq 0$ is a sufficient condition for the pair $\left(\theta^{*}, \theta^{*}\right)$ to be an equilibrium.

Proof: see Ronnen (1991).

Using Assumption 1, the couple of qualities satisfying Ronnen's theorem is $\left(\theta^{*}{ }_{H}=.2533 a^{2}, \theta^{*}{ }_{L}=.0482 a^{2}\right)$.

However, this couple indeed forms an equilibrium only if: (a) inequality (5) is satisfied and (b) none of the duopolists have incentives to deviate and set a value for quality which would bring the two firms to play the price subgame in a different market configuration. Condition (b) will be discussed later, while it is easy to verify that (a) is true only when $d_{p} \geq 4.70$.

\subsection{Full market coverage}

If an equilibrium exists in the covered configuration it is unique and satisfies the principle of maximum differentiation. In fact, the low-quality firm's profits are

$$
\pi_{L}=\frac{(a-2)^{2}\left(\boldsymbol{\theta}_{H}-\boldsymbol{\theta}_{L}\right)}{9}-C\left(\boldsymbol{\theta}_{L}\right) .
$$

Clearly, they are decreasing in $\theta_{L}$, so that the latter will be set at the lower value that satisfies (9), that is

$$
\boldsymbol{\theta}_{L}\left(\boldsymbol{\theta}_{H}\right)=\frac{2-a}{2 a-1} \boldsymbol{\theta}_{H}
$$

The profit function for the high-quality firm is $\pi_{H}=\frac{(a+1)^{2}\left(\boldsymbol{\theta}_{H}-\boldsymbol{\theta}_{L}\right)}{9}-\frac{1}{2} \theta_{H}^{2}$, globally concave and with a unique maximum that doesn't depend on $\theta_{L}$. The equilibrium quality candidates are then:

$$
\left(\boldsymbol{\theta}^{* *}{ }_{H}=(a+1)^{2} / 9, \boldsymbol{\theta}^{* *} *_{L}=\frac{(a+1)^{2}(2-a)}{9(2 a-1)}\right)^{19}
$$

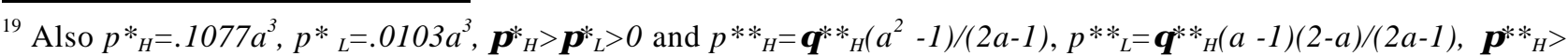
$\pi^{*} *_{L}$. Clearly, this last result would call for a study of entry in the market. We will avoid this problem during this analysis; however, it seems likely that the results of Shaked and Sutton (1982 and 1983) on natural duopolies for the covered configuration and the corner solution can be extended to the case of quality-dependent fixed costs. In the uncovered configuration, a firm can always enter the market with very low quality and obtain positive profits. Thus, under these assumptions, the only possible equilibrium has infinitely many rivals in the market. However if we modify the structure of total costs: $\mathrm{TC}=\mathrm{C}(\mathrm{q})+\mathrm{c}$, where $\mathrm{c}$ is some "small" fixed entry cost, only finitely many competitors enter the market, all with different qualities. The characteristics of the equilibrium are qualitatively identical to the ones shown in the paper. On this point, see Ronnen (1991).
} 
Notice however that, contrarily to the previous case, both firms might suffer negative equilibrium profits, so that in the first stage of the game one or both firms might stay out of the market.

In fact, while no restrictions have to be imposed on $d_{p}$ to satisfy (9), it is easy to verify that $\partial \pi_{L}^{* *} / \partial d_{p}<0$, and that $\pi^{* *}{ }_{L}<0$ if $d_{p}>2.93$ : for sufficiently high values of $d_{p}$, the equilibrium would involve only one firm entering the market and behaving like a monopolist.

\subsection{The corner solution}

As for the corner market configuration, both firms' profits are continuous and always concave ${ }^{20}$. The unique candidate for an equilibrium, $\left(\theta_{H}^{C}, \theta_{L}^{C}\right)$ is the unique non-complex solution of the following thirdorder system of FOCs:

$$
\left\{\begin{array}{l}
\frac{\left(a \boldsymbol{\theta}_{H}-\boldsymbol{\theta}_{L}\right)\left(a \boldsymbol{\theta}_{H}-2 a \boldsymbol{\theta}_{L}+\boldsymbol{\theta}_{L}\right)}{\left(\boldsymbol{\theta}_{H}-\boldsymbol{\theta}_{L}\right)^{2}}-\boldsymbol{\theta}_{H}=0 \\
-\frac{(a-1)\left(a \boldsymbol{\theta}_{H}^{2}+2 \boldsymbol{\theta}_{H} \boldsymbol{\theta}_{L}-\boldsymbol{\theta}_{L}^{2}-\boldsymbol{\theta}_{H}^{2}\right)}{2\left(\boldsymbol{\theta}_{H}-\boldsymbol{\theta}_{L}\right)^{2}}-\boldsymbol{\theta}_{L}=0
\end{array}\right.
$$

which cannot be obtained in closed form. However, using the Theorem of Implicit Functions we can prove that the best response function of the low-quality firm is always increasing and concave while the one for the high-quality firm is concave, first increasing and then decreasing. Also, defining $\theta_{H}=B R_{H}\left(\Theta_{L}\right)$ and $\theta_{L}=B R_{L}\left(\theta_{H}\right)$ as the two (continuous) best response functions, it is easy to show that $B R_{L}(0)<0$, $B R_{L}^{-1}(0)<B R_{H}(0)$. These conditions are indeed sufficient for the existence and uniqueness of this equilibrium candidate ${ }^{21}$.

Similarly to the two previous cases, this couple of qualities forms an equilibrium in a corner configuration only if (a) inequalities (10) are satisfied and (b) none of the duopolists have incentives to deviate and set a value for quality which would bring the two firms to play the price subgame in a different market

\footnotetext{
${ }^{20}$ For general, well defined cost functions, an interior solution in a corner configuration for the quality choice actually exists only if marginal costs are sufficiently steep, that is $\frac{\partial^{2} \pi_{H}^{C}}{\partial \boldsymbol{\Theta}_{H}^{2}} \leq 0$ if and only if $M C^{\prime}\left(\boldsymbol{\Theta}_{H}\right) \geq \frac{\boldsymbol{\Theta}_{L}^{2}(a-1)^{2}}{2\left(\boldsymbol{\Theta}_{H}-\boldsymbol{\Theta}_{L}\right)^{3}}$. This condition is satisfied by Assumption 1.

${ }^{21}$ Also, profits are always positive for both firms (see footnote 17).
} 
configuration. As for part (a), it is simply a matter of computation to show that while the equilibrium candidates always satisfy (10) for low values of $d_{p}\left(d_{p}=2\right.$ included), this is not true when $d_{p}>4.73$. However, an equilibrium may still exist where the two qualities are optimally set such that $\theta_{L}^{C} /(4-3 a)=\theta_{H}^{C}$, that is when the first part of (10) is satisfied as an equality. In this case, there is in fact no incentive to deviate inside the covered configuration for neither firms, even if the optimal choice for the low-quality firm is positive only if $d_{p}<10$. Then, the (unique) solution of the system (15) is a candidate for an equilibrium only when $10<d_{p}<2$.

Also, notice that $\theta^{* *}{ }_{L}$ corresponds to the upper bound for the low-quality firm that defines a corner solution ${ }^{22}$. Then, for a given $\theta^{* *}{ }_{H}$, the low-quality firm's best-response in the covered configuration will be always beaten by the one in the corner solution, whenever this one is defined. As we noticed earlier, the assumption on the cost function limits the existence of an equilibrium with two operative firms in the covered configuration to values of $d_{p}$ such that $d_{p}<2.93$. This has the following implication:

Proposition 3 Quality-dependent fixed costs might exclude the existence of a duopoly equilibrium in a covered configuration where firms move simultaneously. This is the case when $C(\theta)=\frac{1}{2} \theta^{2}$.

In Wauthy (1996) the absence of any cost for the investment in quality created exactly the opposite situation. Contrarily to our case, there it existed a range for the parameter $d_{p}$ such that only the covered configuration equilibrium was defined and an equilibrium there existed. Here, the equilibrium profits of the low-quality firm depends negatively on $d_{p}$ in both configurations, but the corner solution candidate is defined for a wider range of values for $d_{p}$, so that it always creates an incentive for the low-quality firm to deviate if it happens to be in a covered configuration.

In any case, it is noteworthy that the transition from uncovered market structures to covered ones is not smooth. The nature of competition changes when the market is covered; in that case price competition becomes a pure battle for market shares. No a priori condition should be imposed.

\footnotetext{
${ }^{22}$ The second inequality of (10) can in fact be rewritten as $\boldsymbol{\Theta}_{L} \leq \boldsymbol{\Theta}_{H} \frac{2-a}{2 a-1}$. On this point, see also Lambertini and Ecchia (1998).
} 


\subsection{The subgame perfect equilibrium}

The analysis in subsections $4.2,4.3$ and 4.4 simply established that a subgame perfect equilibrium in qualities can exist in an uncovered configuration or in a corner solution, and that for a range of $d_{p}$ $\left(4.70<d_{p}<10\right)$ both candidates are defined. In order to find the subgame perfect equilibrium, however, we still need to check whether one of the two players (or both) wants to deviate to another configuration, given what the opponent does.

Considering first $\left(\boldsymbol{\theta}^{*}, \boldsymbol{\theta}^{*}\right)$, we can in fact prove that for some values of $d_{p}$ the high-quality firm wants to deviate to the corner solution. Given $\theta^{*}=.0482 a^{2}$, this firm can fix

$$
.0482 a^{2} \frac{2 a-1}{2-a} \leq \theta_{H}^{D} \leq \frac{.0482 a^{2}}{4-3 a},{ }^{23}
$$

to maximize $\pi_{H}^{D}=\pi_{H}^{C}\left(\boldsymbol{\theta}_{H}, \boldsymbol{\theta}_{L}^{*}\right)$. This is indeed a profitable deviation if $a<4.76$. In particular if $4.72<d_{p}<4.76, \boldsymbol{\theta}_{H}^{D}=\frac{.0482 a^{2}}{4-3 a}$, while if $4.70<d_{p}<4.72$ the optimal deviation is an interior solution in the interval (16). No other deviation is profitable for neither firms ${ }^{24}$, so in conclusion a subgame perfect equilibrium exists in an uncovered configuration only if $d_{p}>4.76$.

Considering the corner solution, we limit first our analysis to the case $d_{p}>4.73$, so that $\theta_{L}^{C}$ satisfies the first part of (10) as an equality. Suppose for instance $\theta_{H}^{C}=\theta_{L}^{C} /(4-3 a)$, so that $\theta_{L}^{C}=a / 2-5 / 9$. In this case, even if the high-quality firm has no incentive to deviate inside the configuration by setting an interior optimum, some algebra shows that its profits are always higher in an uncovered configuration, that is $\pi_{H}^{D}=\pi_{H}^{*}\left(\boldsymbol{\theta}_{H}^{D}, \boldsymbol{\theta}_{L}^{C}\right)>\pi_{H}^{C}\left(\boldsymbol{\theta}_{H}^{C}, \boldsymbol{\theta}_{L}^{C}\right)$ for any $d_{p}$ in the relevant interval ${ }^{25}$.

When $2<d_{p}<4.73$ a subgame perfect equilibrium may exist only in the corner configuration. However, while there is no incentive to the low-quality firm to deviate in the uncovered configuration (even with leapfrogging), when $4.72<d_{p}<4.73$ the high-quality firm would find profitable to deviate in the uncovered

\footnotetext{
${ }^{23}$ So that condition (9) is satisfied. $D$ stands for deviation. Note that for this deviation to be possible $d_{p}>4$, but we are clearly in this case.

${ }^{24}$ Including leapfrogging in another configuration.

${ }^{25}$ The same thing would happen if the first part of (10) would be set as an equality for the low-quality firm. In this case, $\boldsymbol{\theta}_{L}^{C}=\theta_{H}^{C}(4-3 a)$, where then $\Theta_{H}^{C}=2 a / 3$-4/9. Again, it happens that $\pi_{L}^{D}=\pi_{L}^{*}\left(\theta_{H}^{C}, \theta_{L}^{D}\right)>\pi_{L}^{C}\left(\theta_{H}^{C}, \theta_{L}^{C}\right)$.
} 
configuration with the optimal choice of $\theta_{H}^{P_{H}}$ which satisfies (5) given $\theta_{L}^{C}$ : $\pi_{H}^{D}=\pi_{H}^{*}\left(\boldsymbol{\theta}_{H}^{D}, \boldsymbol{\theta}_{L}^{C}\right)>\pi_{H}^{C}\left(\boldsymbol{\theta}_{H}^{D}, \boldsymbol{\theta}_{L}^{C}\right)^{26}$. In conclusion:

Proposition 4 If $d_{p}>4.76$ a unique subgame perfect equilibrium exists where both firms operate in an uncovered market configuration. If $4.72<d_{p}<4.76$ no pure strategy subgame perfect equilibrium exists. If $2<d_{p}<4.72$ a unique subgame perfect equilibrium exists with both firms operating in a corner solution. If $\mathrm{C}(\theta)=\frac{1}{2} \theta^{2}$ a subgame perfect equilibrium in a covered market configuration doesn't exists.

\section{Welfare analysis}

The equilibrium in qualities is always suboptimal. As we will see, the welfare-maximizing values $\left(\theta_{L}, \theta_{H}\right)^{\mathrm{W}}$ are always greater than the ones emerging from an "unregulated" equilibrium, even if it is not clear a priori whether the social optimum calls for more or less quality differentiation: the trade-off would be of course between higher qualities and lower prices. It is important to notice, however, that if the market configuration is endogenous to the quality choice of the duopolists, it is not obvious that the social optimum has to be found in the same market configuration of the equilibrium. To prove this point, we define the social optimum as the sum of consumer surplus and producer surplus (the latter being the sum of the two firms' profits); in other words:

$$
W=\int_{\underline{S}}^{\frac{p_{H}-p_{L}}{\theta_{H}^{-\theta_{L}}}} t \boldsymbol{\Theta}_{L} d t+\int_{\frac{p_{H}-p_{L}}{\theta_{H}-\theta_{L}}}^{a} t \boldsymbol{\Theta}_{H} d t-\frac{1}{2}\left(\boldsymbol{\theta}_{L}^{2}+\boldsymbol{\Theta}_{H}^{2}\right)
$$

where $\underline{s}=p_{L} / \theta_{L}$ if the market is uncovered, otherwise $\underline{s}=a-1$. Prices are the usual ones emerging from the Bertrand competition in the third stage of the game in a particular market configuration. ${ }^{27}$

\footnotetext{
${ }^{26}$ Finally, note that a monopoly can never be an equilibrium with quality dependent fixed costs. A monopolistic firm in this market would always operate in an uncovered configuration. In fact, its total revenues, $T R_{M}=p\left(a-p / \theta_{M}\right)$, are maximized in the third stage by $p_{M}=a \boldsymbol{\Theta}_{M} / 2$ and this brings a total market share strictly less than one, as required. The quality choice in the second stage would be $\Theta_{M}=a^{2} / 4$, but it exists an $d_{p} *>2$ such that the lowquality firm can profitably deviate and entering with positive profits in an uncovered situation if $d_{p}>d_{p} *$ or in a corner solution if $2<d_{p}<d_{p} *$. On this point also see Ronnen (1991) and Wauthy (1996)

${ }^{27}$ We are then considering the decision of a policy-maker who cannot control directly the price competition of the two firms, but only quality investments.
} 
In order to find the socially optimal market configuration for different values of $d_{p}$ we will follow a similar procedure to the one used for market equilibrium. First, we will find the welfare-maximizing choice of qualities conditional on a particular market configuration. Second, we check if for particular intervals of the index $d_{p}$ more than one socially optimal couple of qualities is defined. Third, if this happens we compare the total welfare of these two or more values to define the socially optimal couple of qualities.

For all market configurations, the maximization problem is the following ${ }^{28}$ :

$$
\begin{aligned}
& \max _{\theta_{L}, \boldsymbol{\theta}_{H}} W=W\left(\boldsymbol{\theta}_{L}, \boldsymbol{\theta}_{H}\right) \\
& \text { s.t. 1) } \boldsymbol{\theta}_{H}>\boldsymbol{\theta}_{L} \geq 0 \\
& \text { 2) } \pi_{H}, \pi_{L} \geq 0
\end{aligned}
$$

In the uncovered configuration, constraint (2) is actually binding for the low-quality firm, so that $\pi^{W *}{ }_{L}=0^{29}$. The socially optimal choice would then be $\left(\theta^{W *}{ }_{H}=.37859 a^{2}, \theta^{W *}{ }_{L}=.10443 a^{2}\right)$. Notice that both qualities are higher than in the uncovered equilibrium; however, their ratio $d_{q}$ decreases. Since $\frac{\partial p_{L}^{*}}{\partial d_{q}}, \frac{\partial p_{H}^{*}}{\partial d_{q}}>0$, the welfare optimum requires, as expected, higher qualities but lower differentiation, in order to make price competition tougher in the third stage. The resulting maximized welfare becomes:

$W^{*}=.080821 a^{4}$

This solution however is defined only if (5) is satisfied, that is if $d_{p} \geq 5.14$. When $d_{p}$ is lower, a policy-maker needs to maximize social welfare in another market configuration. If the market is covered, welfare becomes:

$\frac{-5 a^{2} \boldsymbol{\theta}_{L}+14 a \boldsymbol{\theta}_{L}-8 \boldsymbol{\theta}_{l}+5 a^{2} \boldsymbol{\theta}_{H}+4 a \boldsymbol{\theta}_{H}-\boldsymbol{\theta}_{H}}{18}-\frac{1}{2}\left(\boldsymbol{\theta}_{H}^{2}+\boldsymbol{\theta}_{L}^{2}\right)$

where the sum of the first three terms of the numerator is negative for $d_{p}>2$, implying that the principle of maximum differentiation for the low-quality firm [eq.(13)] holds here too. In this case

$\theta_{H}^{W * *}=\left(5 a^{2}+4 a-1\right) / 18>\theta^{* *}{ }_{H}$,

and while the distance is obviously constant, both qualities increase. The resulting maximized welfare is:

\footnotetext{
${ }^{28}$ In other words, the social optimum is defined as the couple $\left(\boldsymbol{\theta}_{H}^{W}, \boldsymbol{\theta}_{L}^{W}\right)$ that maximizes social welfare if in the final stage of the game, price competition is unregulated.

${ }^{29}$ The welfare function in this case is $W^{*}=\frac{1}{2} a^{2} \boldsymbol{\theta}_{H} \frac{-\boldsymbol{\theta}_{H} \boldsymbol{\theta}_{L}-2 \boldsymbol{\theta}_{L}^{2}+12 \boldsymbol{\theta}_{H}^{2}}{\left(4 \boldsymbol{\Theta}_{H}-\boldsymbol{\theta}_{L}\right)^{2}}-\frac{1}{2}\left(\boldsymbol{\Theta}_{L}^{2}+\boldsymbol{\theta}_{H}^{2}\right)$.
} 


$$
W^{* *}=\frac{-591 a^{2}+288 a-330 a^{5}+175 a^{6}-35+50^{3}+459 a^{4}}{648(2 a-1)^{2}}
$$

However, constraint (2) is not binding for the low-quality firm only when $d_{p} \leq 2.42$. Otherwise, the socially optimum quality choice becomes $\left(\theta^{W * *}{ }_{H}=2(2 a-1)(a-1) / 3, \quad \theta^{W * *}{ }_{L}=\left(6 a-4-2 a^{2}\right) / 3\right)$ and the resulting maximized welfare function is:

$$
\left.W^{* * *}\right|_{\pi^{* *}=0}=\frac{-5 a^{4}+8 a^{3}-35 a^{2}+21 a-5}{9}
$$

A possible social optimum is also available in the corner solution. In this case, we are unable to obtain a closed form solution for $\left(\theta_{\mathrm{L}}^{\mathrm{WC}}, \theta_{\mathrm{HC}}^{\mathrm{WC}}\right)$; however, it is always optimal for a social planner to set $\pi^{C}{ }_{L}=0$. In other words,

$$
\theta_{H}^{C}=\theta_{L}^{C} \frac{1-a+\theta_{L}^{C}}{a^{2}-3 a+2+\theta_{L}^{C}} \cdot{ }^{30}
$$

At its turn, condition (23) implies that there might be values of $d_{p}$ such that condition (10) will not be satisfied at the optimum, otherwise profits for the low-quality firm would become negative again. In fact, it is just a matter of algebra to show that a possible social optimum is in fact defined only when $2.47<d_{p}<5.15$.

Finally, comparing total welfare in (18), (21) and the one for the corner solution for the range of $d_{p}$ such that more than one possible social optimum is defined, we reach the following conclusion:

Proposition 5 i) if $d_{p}>5.15$, a social optimum is defined in both covered and uncovered configuration. The uncovered market configuration will offer a greater total surplus; ii) if $5.14<d_{p}<5.15$, a social optimum is defined in all configurations. The corner configuration will offer a greater surplus; iii) if $2.47<d_{p}<5.14$, a social optimum is defined in both covered and corner configuration. The corner solution will offer a greater total surplus; iv) if $2.42<d_{p}<2.47$, a social optimum is defined only in the covered configuration and both firms enjoys positive profits; v) if $2<a<2.42$ a social optimum is defined only in the covered configuration

\footnotetext{
${ }^{30}$ See the expression for $T R_{L}^{C}$ at the beginning of section 3 .
} 
and the profits for the low quality firm are equal to 0 . Also, the unregulated equilibrium configuration doesn't always correspond with the welfare-maximizing market configuration. ${ }^{31}$

\section{Conclusions}

In our analysis we investigated the existence of a pure-strategy subgame perfect equilibrium in a vertically differentiated duopolies with fixed costs of quality improvements. Contrarily to the analysis conducted by Wauthy (1996), where firms produce at no cost, the high-quality firm has now an incentive to decrease quality in order to reduce production costs and/or increase its market share. Hence, a pure-strategy subgame perfect equilibrium may fail to exist. This fact was also recognized by Lambertini and Ecchia (1998), who assumed variable costs for quality improvements.

In this aspect, also models of vertical differentiation with the finiteness property suffer from a problem of non-existence of the equilibrium in pure strategies, similarly to what happens with horizontally differentiated duopolies.

However, differently from that contribution, the degree of convexity of fixed quality production costs in the relevant parameter space doesn't prevent a unique equilibrium with a corner solution to exist. What disappears, given our assumption on fixed costs, is a subgame perfect equilibrium in which the market is fully covered.

Finally, we showed that models with vertical differentiation should be carefully used for policy analysis. The welfare comparison between the equilibrium and the social optimum should be performed without assuming ex-ante a full or partial coverage of the market. For some parameter range, the socially optimal configuration doesn't coincide with the one emerging in the unregulated equilibrium.

\footnotetext{
${ }^{31}$ For instance, when $5.14<d_{p}<5.15$ the unique market equilibrium is in the uncovered configuration, while the social optimum is in the corner solution. Also, when $a<2.47$ the equilibrium is in the corner solution, while the social optimum is maximized in a covered configuration.
} 


\section{REFERENCES}

Choi, J.C. and Shin, H.S. (1992), “A Comment on a Model of vertical Product Differentiation”, Journal of Industrial Economics, v.40, 229-31.

Constantatos, C. and Perrakis, S. (1998), "Minimum Quality Standards, Entry and the Timing of the Quality Decision", Journal of Regulatory Economics, v.13, 47-58. 
Crampes, C. and Hollander, A. (1995), "Duopoly and Quality Standards". European Economic Review, v.39, 71-82.

Gabszewicz, J.J. and Thisse, J.-F. (1979), "Price Competition, Quality and Income Disparities”, Journal of Economic Theory, v.20, 340-359.

Ecchia, G. and Lambertini, L. (1998), "Market Coverage and the Existence of Equilibrium in a Vertically Differentiated Duopoly”, Working Paper, Department of Economics, University of Bologna, 311/21.

Mussa, M. and Rosen, S. (1978), "Monopoly and Product Quality”, Journal of Economic Theory, v.18, 301-317.

Polo, M. (1991), Mercati, varietà e informazione: un'analisi teorica, Egea, Milano.

Ronnen, U. (1991), "Minimum Quality Standard, Fixed Costs and Competition", Rand Journal of Economics, v.22, 490-504.

Shaked, A. and Sutton, J. (1982), "Relaxing Price Competition through Product Differentiation”, Review of Economic Studies, v.69, 3-13.

Shaked, A. and Sutton, J. (1983), "Natural Oligopolies”, Econometrica, v.51, 1469-83.

Tirole, J. (1988), The Theory of Industrial Organization, Cambridge, MA, MIT Press.

Wauthy, X. (1996), "Quality Choice in Models of Vertical Differentiation”, Journal of Industrial Economics, v.44, 345-53. 